\title{
144. On a Theorem on Function Space of A. Grothendieck
}

\section{By Kiyoshi Iś́KI}

(Comm. by K. Kunugi, M.J.A., Dec. 12, 1957)

Very interesting results on countable compactness of function spaces have been obtained by $\mathrm{A}$. Grothendieck [3]. In this paper, we shall consider the case of a set of real valued continuous functions on a pseudo-compact space, and we shall give a generalisation of his result. Let $E$ be a pseudo-compact space, and let $C_{s}(E)$ be the topological space of all continuous functions on $E$ with simple convergence topology. Then if a subset $A$ of $C_{s}(E)$ is conditionally compact, then it is conditionally countably compact. Next, if $A$ is conditionally countably compact, for every sequence $\left\{f_{m}\right\}$ of $A$ and countable set $\left\{x_{m}\right\}$ of $E$, there is a continuous function $f(x)$ on the closure $C$ of $\left\{x_{n}\right\}$ such that, for every $x$ of $C, f(x)$ is a cluster point of $f_{n}(x)$ and $\left\{f_{n}(x)\right\}$ is pointwise bounded.

Let $\left\{f_{m}\right\}$ be a countable set of $C_{s}(E)$, and let $\left\{x_{n}\right\}$ be a countable set of $E$. Then following A. Grothendieck [3] we shall define a double cluster point $\alpha$ of the double sequence $\left\{f_{m}\left(x_{n}\right)\right\}$ as follows.

A point (number) $\alpha$ is said to be a double cluster point of $\left\{f_{m}\left(x_{n}\right)\right\}$, if, for each neighbourhood $U$ of $\alpha$, and a given integer $N$, there are infinitely many $f_{m}\left(x_{n}\right)$ meeting $U$ for $m, n \geq N$.

If $\left\{f_{m}\right\}$ and $\left\{x_{n}\right\}$ satisfy the conditions in the previous section, then $\left\{f_{m}\left(x_{n}\right)\right\}$ has at least one double cluster point. To prove it, we shall define an equivalent relation on $E$. For $x, y$ of $E$, we define $\rho(x, y)$ by

$$
\rho(x, y)=\sum_{n=1}^{\infty} \frac{1}{2^{n}} \frac{\left|f_{n}(x)-f_{n}(y)\right|}{1+\left|f_{n}(x)-f_{n}(y)\right|}
$$

(for example, see R. G. Bartle [1, p. 48]).

By $\rho(x, y)=0$ we shall define an equivalent relation $x \sim y$. Then the space $E$ is decomposed into equivalent classes by the relation " ". By $\&$, we denote the set of equivalent classes. Then $\mathbb{E}$ is a metric space with the metric $\rho$. By the continuity of the natural mapping $F: E \rightarrow \mathbb{E}$, E is a compact metric space with respect to $\rho$ and $X^{\mathrm{p}}=F(x)$ is the class containing $x$. For $f \in C_{s}(E)$, we shall define $\varphi$ on $\&$ by $\varphi\left(X^{p}\right)=$ $f(x)$, where $X^{\mathrm{p}}=F(x)$. Therefore, for $f_{m}$ and $f$, we have continuous functions $\varphi_{n}\left(X^{\rho}\right), \varphi\left(X^{\rho}\right)$ on $X^{\mathrm{p}}$ for $x \in C$. Since $\&$ is compact, ${ }^{1)}$ the set $X^{p}$ for $x$ of $C$ has at least one cluster point $X_{0}^{p}$. Therefore let $\alpha$ be $\varphi\left(X_{0}^{\mathrm{p}}\right)$, then we obtain that $\alpha$ is a double cluster of $f_{m}\left(x_{n}\right)$. It is sufficient to show that $\varphi\left(X_{0}^{\mathrm{p}}\right)$ is a double cluster point of $\varphi_{m}\left(X_{n}^{\mathrm{p}}\right)$, where $X_{n}^{\mathrm{p}}=F\left(x_{n}\right)$. Since $X_{0}^{\mathrm{p}}$ is a cluster point of $\left\{X_{n}^{\mathrm{\rho}}\right\}$, there is a subsequence $\left\{X_{n_{i}}^{\mathrm{\rho}}\right\}$ which

1) See. K. Iséki 「5. o. 4247. 
converges to $X_{0}^{\rho}$, we have $\varphi_{m}\left(X_{n_{i}}^{\rho}\right) \rightarrow \varphi_{m}\left(X_{0}^{p}\right)$ for every $m$.

On the other hand, since $\varphi\left(X^{p}\right)$ is continuous, $\varphi_{m}\left(X^{p}\right)$ is simply uniformly convergent to $\varphi\left(X^{p}\right)$. Hence, for every $\varepsilon>0$ and every $M$, there is an integer $m \geq M$ and a neighbourhood $U$ of $X_{0}^{p}$ such that

$$
\left|\varphi_{m}\left(X^{p}\right)-\varphi\left(X^{p}\right)\right|<\varepsilon
$$

for $X^{p} \in U$. Therefore, for infinitely many $n$, we have

$$
\left|\varphi_{m}\left(X_{n_{i}}^{p}\right)-\varphi\left(X_{n_{i}}^{p}\right)\right|<\varepsilon
$$

Since $\varphi\left(X_{n_{i}}^{\rho}\right) \rightarrow \varphi\left(X_{0}^{p}\right)$, we have $\varphi_{m}\left(X_{n_{i}}^{\rho}\right) \rightarrow \varphi\left(X_{0}^{p}\right)$ for $i \rightarrow \infty$. Next we must show that there are infinitely many $n$ and for the every fixed $n$, each neighbourhood of $\varphi\left(X_{0}^{p}\right)$ meets infinitely many of $\varphi_{m}\left(X_{n}^{p}\right)$. For a given $\varepsilon>0$, we can find a neighbourhood $U$ of $X_{0}^{p}$ such that $\left|\varphi\left(X^{p}\right)-\varphi\left(X_{0}^{p}\right)\right|$ $<\varepsilon$ for $X^{p} \in U$. Since $U$ contains infinitely many of $X_{n}^{\mathrm{p}}$, we shall take one point $X_{n_{i}}^{\rho}$ of $\left\{X_{n}^{\rho}\right\}$. Then we can take an integer $M$ such that $M \leq m$ implies

and, hence, we have

$$
\left|f_{m}\left(X_{n_{i}}^{\mathrm{p}}\right)-f\left(X_{n_{i}}^{\mathrm{p}}\right)\right|<\varepsilon
$$

$$
\left|\varphi_{m}\left(X_{n_{i}}^{\mathrm{p}}\right)-\varphi\left(X_{0}^{\mathrm{p}}\right)\right| \leq\left|\varphi_{m}\left(X_{n_{i}}^{\mathrm{p}}\right)-\varphi\left(X_{n_{i}}^{\mathrm{p}}\right)\right|+\left|\varphi\left(X_{n_{i}}^{\mathrm{p}}\right)-\varphi\left(X_{0}^{\mathrm{p}}\right)\right|<\mathbf{2} \varepsilon
$$

for $M \leq m$. Therefore, the proof is complete.

We shall show that the condition concluded and the pointwise boundedness imply the conditionally compactness of $A$. To prove it, we use the technique of F. Eberlein. ${ }^{2}$ The available method was also used by A. Grothendieck ([3, p. 173] or $[4$, p. 19]). Therefore, our proof is similar with them. For each $x$, let $l_{x}$ be $\max _{f \in A}|f(x)|$, then $l_{x}$ is finite. Next consider the product space $\prod_{x \in E}\left[-l_{x}, l_{x}\right]$ with weak topology, where $\left[-l_{x}, l_{x}\right]$ denotes the interval $\left\{y \mid-l_{x} \leq y \leq l_{x}\right\}$. By Tychonov theorem, the product space is compact, and $A$ is considered as a subset of the space. We shall prove that an element of the closure $\bar{A}$ of $A$ is a continuous function. ${ }^{3)}$ Suppose that $f(x)$ is a non-continuous function of $\bar{A}$, then there is a point $x_{0}$ of $E$ such that $f(x)$ is not continuous at $x_{0}$. Therefore there is a positive number $\varepsilon$ such that for every neighbourhood $U$ of $x_{0}$, we can find a point $x$ of $U$ satisfying $\left|f(x)-f\left(x_{0}\right)\right| \geq \varepsilon$. We define $f_{n}(x)$ of $A$ and $x_{n}$ of $E$ by the following relations recursively.

$$
\begin{array}{ll}
\left|f_{n}\left(x_{i}\right)-f\left(x_{i}\right)\right| \leq \frac{1}{n} & \text { for } \quad 0 \leq i \leq n-1 \\
\left|f_{i}\left(x_{n}\right)-f_{i}\left(x_{0}\right)\right| \leq \frac{1}{n} & \text { for } \quad 0 \leq i \leq n
\end{array}
$$

and

$$
\left|f\left(x_{n}\right)-f\left(x_{0}\right)\right| \geq \varepsilon
$$

This is possible by the hypothesis. Let $\alpha$ be a double cluster point of $\left\{f_{m}\left(x_{n}\right)\right\}$. From these relations, we have $f_{n}\left(x_{i}\right) \rightarrow f\left(x_{i}\right)(n \rightarrow \infty)$ for every

2) See, F. Eberlein: Weak compactness in Banach spaces I, Proc. Nat. Acad. Sci. U. S. A., 33, 51-53 (1947); or A. Grothendieck [4].

3) On convergence, filter, see G. Bruns und J. Schmidt [2]. 
$i$, and $f_{i}(x) \rightarrow f_{i}\left(x_{0}\right)(n \rightarrow \infty)$. Hence $\alpha$ is a cluster point of $\left\{f\left(x_{i}\right)\right\}$ and $\left\{f_{m}\left(x_{0}\right)\right\}$, and by (1), $f_{m}\left(x_{0}\right) \rightarrow f\left(x_{0}\right)$. Therefore we have $a=f\left(x_{0}\right)$, which contradicts (3). Hence $f(x)$ is continuous at $x_{0}$ therefore we have the following

Theorem 1. Let $E$ be a pseudo-compact space, and $A$ a subset of $C_{s}(E)$, then the following conditions are equivalent:

1) $A$ is conditionally compact.

2) $A$ is conditionally countably compact.

3) $A$ is pointwise bounded, and for every sequence $f_{n}$ of $A$ and every countable set $x_{n}$ of $E$, there is a continuous function $f(x)$ on the closure $C$ of $\left\{x_{n}\right\}$ such that, for every $x$ of $C, f(x)$ is a cluster point of $f_{n}(x)$.

4) $A$ is pointwise bounded, and for every sequence $f_{m}$ of $A$ and for every countable set $x_{n}$ of $E, f_{m}\left(x_{n}\right)$ has at least one double cluster point.

Theorem 2. Let $E$ be a topological space, and let $f_{m}(x)$ be any sequence of distinct continuous functions on $E$. Suppose that $f_{m}(x)$ is pointwise bounded, and for every set of countable points, $\left\{f_{m}\left(x_{n}\right)\right\}$ has at least one double cluster point. Then every continuous function on $E$ is bounded.

Proof. If there is an unbounded continuous positive function $f(x)$, then we can find a sequence $\left\{x_{n}\right\}$ of points such that $f\left(x_{n}\right) \geq n$. Then $f_{m}(x)=\operatorname{Min}\{m, f(x)\}(m=1,2, \cdots)$ is a sequence of continuous functions and it is pointwise bounded on $E$. For $m \leq n$ we have

$$
f_{m}\left(x_{n}\right)=m \text {. }
$$

If we denote the $(m, n)$-element by $f_{m}\left(x_{n}\right)$, we have the following infinite matrix

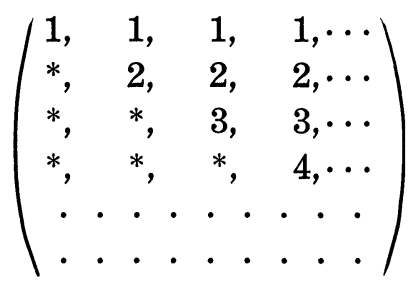

As is easily seen, there is no double cluster point in the double sequence. Therefore the proof is complete.

\section{References}

[1] R. G. Bartle: On compactness in functional analysis, Trans. Am. Math. Soc., 79, 35-37 (1955).

[2] G. Bruns und J. Schmidt: Zur Aquivalenz von Moore-Smith Folge und Filtern, Math. Nachr., 13, 169-186 (1955).

[3] A. Grothendieck: Critères de compacté dans les espaces fonctionnels géréraux, Am. Jour. Math., 74, 168-186 (1952).

[4] A. Grothendieck: Théorie des Espaces Vectoriels Topologiques, Part II, Chap. V (1954) (mimeographed note).

[5] K. Iséki: Pseudo-compactness and strictly continuous convergence, Proc. Japan Acad., 33, 424-428 (1957). 